\title{
Erratum to: "Raman Spectroscopy Study of the Behavior of the Soft Mode in a Structural Phase Transition in the $\operatorname{Pr}_{3} \mathrm{Sb}_{5} \mathrm{O}_{12}$ Crystal" [Physics of the Solid State 57, 2286 (2015)]
}

\author{
A. S. Oreshonkov ${ }^{a, b *}$, A. K. Khodzhibaev ${ }^{c}$, A. S. Krylov ${ }^{a, b}$, M. F. Umarov ${ }^{d}$, and A. N. Vtyurin ${ }^{a, b}$ \\ ${ }^{a}$ Kirensky Institute of Physics, Siberian Branch of the Russian Academy of Sciences, \\ Akademgorodok 50-38, Krasnoyarsk, 660036 Russia \\ ${ }^{b}$ Siberian Federal University, pr. Svobodnyi 79, Krasnoyarsk, 660041 Russia \\ ${ }^{c}$ Khujand State University, pr. Mavlonbekov 1, Khujand, Tajikistan \\ ${ }^{d}$ Vologda State University, ul. Lenina 15, Vologda, 160000 Russia \\ *e-mail: oreshonkov@iph.krasn.ru \\ Received March 15, 2016
}

DOI: $10.1134 / \mathrm{S} 1063783416040351$

The fourth affiliation and e-mail of the corresponding author should read as follows:

d Vologda State University, ul. Lenina 15, Vologda, 160000 Russia

*e-mail: oreshonkov@iph.krasn.ru 